\title{
Office Laboratory Regulations Revisited
}

Eighteen months ago in this editorial column, I reviewed the government's plans for the implementation of the Clinical Laboratory Improvement Amendments of 1988 (P.L. 100-578 or CLIA '88). Since that time, there has been considerable activity to formulate the final regulatory scheme. These regulations were legislated to go into effect in January of 1990, however, to this day, it is still unclear what the final regulations will even look like.

The bill which was passed by Congress in 1988 and signed by the President, regulated all clinical Iaboratories in the United States. A "laboratory" was defined as any site where human specimens were tested for the purpose of diagnosis. The implications of this broad definition were not fully understood at the time that the bill was passed. CLIA regulates all hospital laboratories, commercial laboratories, office laboratories, the research laboratories at medical schools, cholesterol screening programs at malls, the federal laboratories at the Centers for Disease Control $(\mathrm{CDC})$ and the National Institute of Health $(\mathrm{NIH})$, and even the hand-held breathalizers that policemen use to identify intoxicated drivers. If the bill is to be fully implemented, every police patrol car would need to carry a pathologist and every policeman would need to be a certified laboratory technologist!

Congress charged the Health Care Financing Administration (HCFA) with developing the CLIA regulations. In mid 1990, HCFA published its first set of proposed regulations. Most physicians view these proposed regulations as excessive, onerous, impractical and costly. Conservative estimates of the cost of the proposed regulations have ranged between four and ten billion dollars per year. These are costs for solving a problem which may not even exist. There is as yet not a single good study that shows any problem with the quality of testing in U.S. laboratories. We probably have the best laboratory testing in the world. It is clear that given the ballooning costs for health care, CLIA is not money well spent.

The basic structure outlined in the proposed regulation is a three tiered approach based on test complexity. The first level would constitute waivered laboratories. These would be laboratories that confine their testing to simple tests. They would only be required to report their testing to the government every other year. The next level would be for most office laboratories. These laboratories could be directed by any physician and the testing could be done by any office staff. They would be limited to a slightly larger list of simple tests. Finally, the most compex laboratories would need to be directed by a pathologist or clinical chemist and all testing would need to be done by trained technicians or technologists. While conceptionally reasonable, HCFA has made the proposed lists of tests excessively restrictive. In some cases, they have even gone against the wording of the CLIA bill. For example, the CLIA bill says that any test approved by the FDA for over-the-counter use, can be done by a waivered laboratory. However, HCFA has decided that it will permit waivered laboratories only to do visual inspection of whole blood glucose reagent strips, not to use reflectance instruments. This means that any patient can go to their local pharmacy and buy a glucometer for use in their home, but physicians would not be allowed to use these instruments in their office!

Finally, the proposed rules would severely limit patient access to health care. This is especially true in rural areas. There are many 20 bed hospitals in rural America serving populations which have no other access to health care. These hospitals would be unable to provide necessary laboratory services because they could not afford a full-time pathologist laboratory director.

HCFA has received over 50,000 letters in response to their proposed regulations. The most important letter was from the government's Office of Management and Budget (OMB). They have required HCFA to address both the cost of the proposed regulations and its impact on access to care. In addition, HCFA will be required, as originally mandated by Congress, to complete studies on the quality of testing in the United States. These must be completed prior to formulating any final regulations. It is, therefore, very likely that final regulations will not be published until very late in 1991 or in 1992 . Furthermore, there will be a two year grace period for all laboratories before they need to comply with the final regulations. This means that it will be 1993 or 1994 before CLIA has any impact on physicians. Furthermore, with OMB serving in its federal oversight capacity, HCFA will hopefully not be permitted to spend health care dollars to solve problems that do not exist.

Paul M. Fischer, M.D. Associate Professor Department of Family Medicine Medical College of Georgia Augusta, Georgia 30912 